\title{
Topography and axon arbor architecture in the visual callosal pathway: effects of deafferentation and blockade of $N$-methyl-D-aspartate receptors
}

\author{
JAIME F OLAVARRÍA ${ }^{1,2}$, ROBYN LAING ${ }^{1}$, RYOKO HIROI $^{1}$ and JURATE LASIENE ${ }^{1}$
}

\author{
${ }^{1}$ Department of Psychology, University of Washington, Seattle, Washington 98195-1525, U.S.A. \\ ${ }^{2}$ Neurobiology and Behavior Program, University of Washington, Box 351525, Seattle, \\ Washington 98195-1525, U.S.A.
}

\begin{abstract}
Visual callosal fibers link cortical loci in opposite hemispheres that represent the same visual field but whose locations are not mirror-symmetric with respect to the brain midline. Presence of the eyes from postnatal day 4 (P4) to P6 is required for this map to be specified. We tested the hypothesis that specification of the callosal map requires the activation of $N$-methyl-D-aspartate receptors (NMDARs). Our results show that blockade of NMDARs with MK-801 during this critical period did not induce obvious abnormalities in callosal connectivity patterns, suggesting that retinal influences do not operate through NMDAR-mediated processes to specify normal callosal topography. In contrast, we found that interfering with NMDAR function either through MK801-induced blockade of NMDARs starting at P6 or neonatal enucleation significantly increases the length of axon branches and total length of arbors, without major effects on the number of branch tips. Our results further suggest that NMDARs act by altering the initial elaboration of arbors rather than by inhibiting a later-occurring remodeling process. Since the callosal map is present by P6, just as axonal branches of simple architecture grow into gray matter, we suggest that regulation of arbor development by NMDAR-mediated processes is important for maintaining the precision of this map.
\end{abstract}

Key terms: corpus callosum, interhemispheric commissure, map, NMDAR, striate cortex.

\section{INTRODUCTION}

In the cerebral cortex, spatially organized patterns of neural projections, known as topographic maps, are essential for the processing of information in sensory and motor pathways. In the visual cortex, the topographic layout of the retina is represented in each cortical visual area, as well as in the network of orderly projections interconnecting each area with other visual areas located either in the same or opposite hemisphere. The mechanisms guiding the development of these projections are not well understood. Spontaneously generated (Maffei and GalliResta, 1990) and/or sensory-driven neuronal activity in the retina is believed to play an important role in sculpting central circuits from initially imprecise neuronal connections (Katz and Shatz, 1996). Moreover, in many cases the role played by neural activity is mediated by $N$-methyl-Daspartate receptors (NMDARs) (Constantine-Paton et al., 1990; Hahm et al., 1991). Normal retinal input is also required for the normal development of both interhemispheric and intrahemispheric cortico-cortical pathways (see Refs. in Olavarría, 2002). However, the role played by retinal activity and activation of NMDARs on the establishment of corticocortical topography remains poorly understood.

An ideal system for studying mechanisms underlying the development of

Correspondence to: Jaime F. Olavarría, Department of Psychology, University of Washington, Box 351525, Seattle, Washington 98195-1525, U.S.A. Phone: (206) 543-8675, Fax: (206) 685-3157, e-mail: jaime@u.washington.edu 
cortical topographic maps is the system of callosal connections in primary visual cortex (V1, area 17, striate cortex, Fig. 1). Studies in rodents (Lewis and Olavarría, 1995; Olavarría and Hiroi, 2003) have shown that visual callosal fibers interlink opposite cortical loci that are in retinotopic, rather than anatomic, correspondence (i.e., interconnected loci are not mirrorsymmetric with respect to the brain midline, see Fig. 1A, B). Moreover, development of this topography requires retinal input during a brief critical period extending from postnatal day 4 (P4) to P6 (Olavarría and Hiroi, 2003). These authors showed that removal of retinal input at, or prior to $\mathrm{P} 4$ produced abnormal, mirror-symmetric patterns of callosal linkages (Fig. 1C), whereas removal of retinal input at or after P6 resulted in the normal, non-mirror symmetric topography (Fig. 1B, Olavarría and Li, 1995; Olavarría and Hiroi, 2003). Olavarría and Li (1995) proposed that bilateral projections from temporal retina relay synchronous activity to retinotopically corresponding points in both hemispheres, leading to the stabilization of non mirrorsymmetric interhemispheric connections through Hebbian-like (Hebb, 1949) mechanisms (Fig. 1A). Evidence suggesting that NMDARs are involved in callosal development comes from the observation that retinal input during the P4-P6 critical period induces a transient (P6 to P13) increase in the duration of NMDARmediated synaptic currents in callosal cells (Olavarría et al., 2007). In the present study we tested the hypothesis that specification of the callosal map by retinal input during the P4-P6 critical period requires activation of NMDARs. We analyzed the topography of callosal linkages in adult rats following pharmacological blockade of NMDARs during this P4-P6 critical period. Our results show that blockade of NMDARs during this period did not prevent the development of normal callosal topography.

We also investigated whether NMDARs play a role in the elaboration of callosal axon arbors. Evidence that NMDARs regulate the development of axon terminals comes from studies reporting that either blockade or cortex-specific deletion of
NMDARs leads to the development of axonal arbors that are larger than normal (e.g., Brewer and Cotman, 1989; Schmidt et al., 2000; Lee et al., 2005a,b). Invasion of superficial gray matter by visual callosal axons and elaboration of terminal arbors starts by P6 in both normally eyed and neonatally enucleated rats (Olavarría and Safaeian, 2006). We examined the effect that pharmacological blockade of NMDARs starting at P6 has on the morphology of callosal arbors examined in adult rats. In a separate experiment we examined the effect that bilateral enucleation at birth has on the early development of arbor elaboration. In addition to abolishing the transient increase in the duration of NMDAR-mediated synaptic currents that is normally observed from P6 to P13 (Olavarría et al., 2007), enucleation at birth may result in a marked reduction in NMDAR activation because it disrupts the system of bilateral projections from temporal retina that presumably relays synchronous activity to retinotopically corresponding points in both hemispheres. We found that callosal arbors were significantly larger in both enucleated rats and in rats treated with NMDAR blocker starting at P6. Together, the results of this study suggest that normal functioning of NMDARs plays no major role in the specification of callosal maps, but it is necessary for the development of normal callosal arbors.

\section{MATERIALS AND METHODS}

A total of 23 Long-Evans rats were used in this study. The births of the litters were determined to within 12 hours, and the first postnatal day was considered as $\mathrm{P} 0$. To study the effect of NMDAR blockade on the topography of the callosal map, six rats were injected twice daily either from P3 to P7 (one rat) or from P4 to P8 (5 rats) with the NMDA channel blocker (+)-5-methyl10,11-dihydro-5Hdibenzo [a, d] cyclohepten $-5,10$-imine hydrogen maleate (MK-801, 1 mg/Kg each injection, ip), which crosses the blood-brain barrier (MacDonald et al., 1991). To analyze the effect of NMDAR blockade on axonal 

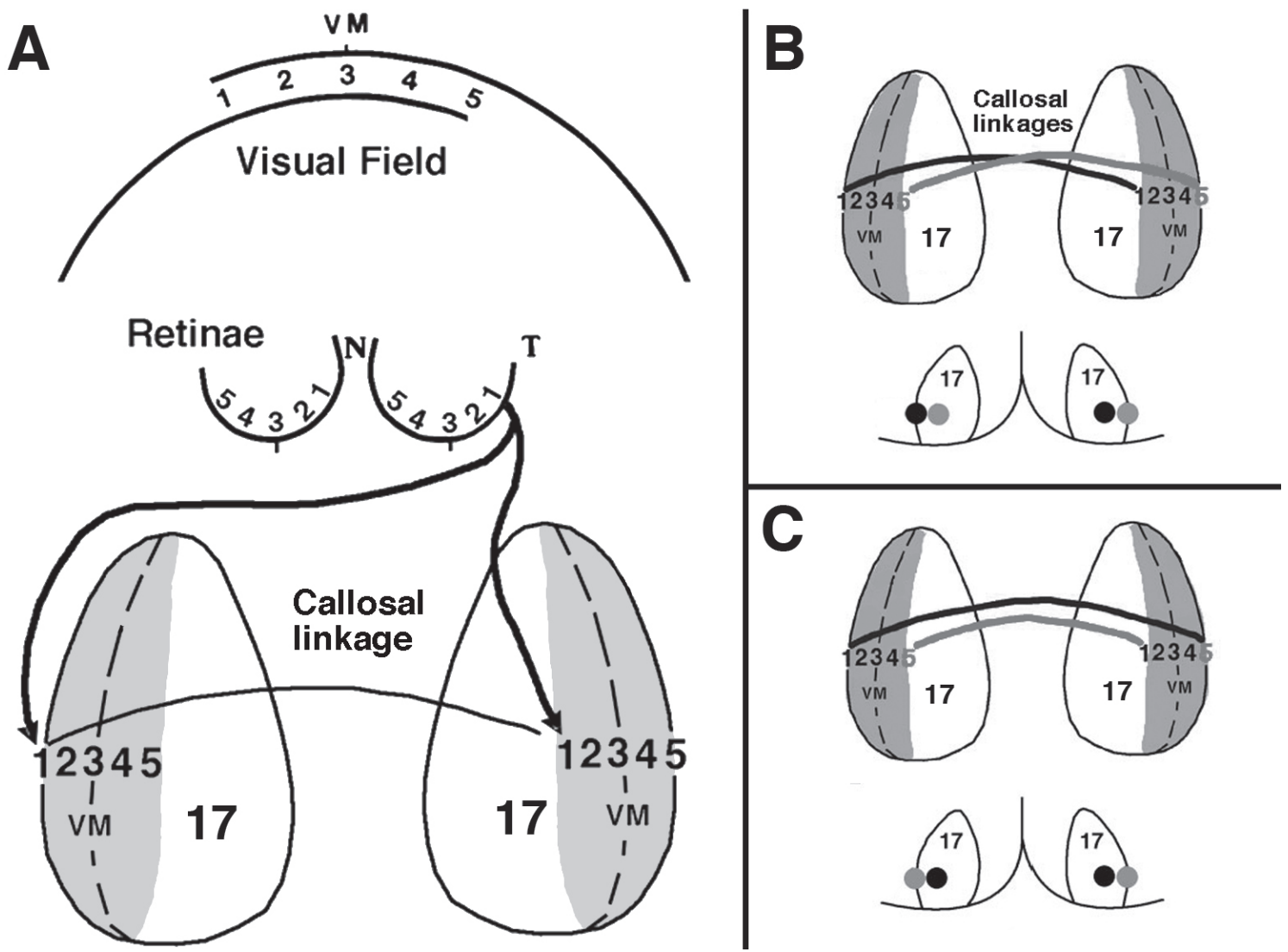

Fig. 1: Schematic diagrams of the topography of callosal linkages in normal and neonatally enucleated rats. Neurons and axon terminals of the callosal pathway accumulate in a column-like zone that straddles the border between area 17 and area 18a (see Olavarría and Hiroi, 2003). Note however that in all diagrams, the areas indicated in gray represent only the portion of the $17 / 18 \mathrm{a}$ callosal zone falling within area 17. A: Diagram illustrating hypothesis that bilateral projections from temporal retina guide the development of retinotopically corresponding callosal linkages in lateral area 17 (striate cortex, V1). The visual field is schematically represented by the semicircular perimeters located at the top of the diagram and the numbers 1 to 5 indicate regularly spaced loci in central regions of the visual field. The vertical meridian of the visual field (VM) is indicated by the locus 3. Only projections from right temporal retina are represented. For simplicity, the dLGN is not included in this diagram. Due to the representation of ipsilateral visual fields in each hemisphere (i.e., segment 3-5 of the visual field is represented in the right V1, and segment 1-3 is represented in the left $\mathrm{V} 1$ ), a central area of the visual field including the vertical meridian is represented in both hemispheres (the visual field segment 1-5). This diagram illustrates that callosal fibers, indicated by the continuous line between the hemispheres, interlink retinotopically corresponding cortical loci (i.e., both the origin and end of callosal fibers represent the same retinal locus). $\mathrm{N}=$ nasal, $\mathrm{T}=$ temporal. (See Lewis and Olavarría, 1995, for additional details.). B: Diagram of non-mirror symmetric (retinotopically corresponding) callosal linkages observed in normal rats. C: Diagram of symmetric (non retinotopically corresponding) callosal linkages in neonatally enucleated rats. In both $\mathrm{B}$ and $\mathrm{C}$, bottom insets illustrate the topography of callosal connections to facilitate comparison with data from this study (Fig. 2). In these diagrams, dots of the same color in opposite hemispheres (gray or black) are connected either non-symmetrically (B) or symmetrically (C) with respect to the brain midline. The dots in the right hemispheres represent tracer injection sites. 
branch development, another group of 3 rats received the same dosage of MK-801 from P6 to P10. Three control pups in each group received injections of equivalent volumes of saline. Five to ten minutes after the MK-801 injections, pups became slightly ataxic and less responsive to manipulation, as previously described in studies using MK-801 (Daw et al., 1999). Four pups (P6-P8) were used to study the effect of neonatal enucleation on the development of callosal arbors. They were anesthetized with halothane $(2-4 \%$ in air) and binocularly enucleated at P0. The data from these animals was compared to results from 4 control pups (P6-P8). All surgical procedures were performed according to protocols approved by the Institutional Animal Care and Use Committee at the University of Washington.

Tracer injections. Tracer injections were made under halothane anesthesia $(2-4 \%$ in air). The analysis of the effect of MK-801 on the topography of the callosal map was performed in animals that were at least 2 months old. Small volumes $(0.05-0.1 \mu \mathrm{l})$ of tracers were injected at various locations into lateral striate cortex of the right hemisphere (approx. 3.7-4.7 $\mathrm{mm}$ from the midline; $0.3-2 \mathrm{~mm}$ anterior to the lambda suture, see Fig. 2). Tracers used included the fluorescent tracers Rhodamine and Green-beads (RB and GB, respectively, LumaFluor, Naples, FL, concentrated stock solution), which are transported retrogradely, and biotinylated dextran amine (BDA, $10 \%$ in DW, Molecular Probes, Eugene, OR), which is predominantly transported anterogradely. These tracers were pressure-injected through glass micropipettes $(50-100 \mu \mathrm{m}$ tip diameter). In all cases analyzed, the small tracer injections used to reveal the callosal map were restricted to gray matter. The effects of MK-801 on the architecture of callosal axon arbors was examined in animals that were at least 2 months old, while the development of callosal arbors in normally reared and neonatally enucleated animals was investigated in pups ranging in age from P6 to P8. Callosal axons and arbors were labeled following multiple (10$20)$ intracortical injections of BDA (10\% in
DW). Adult rats and neonatal pups received about $2 \mu \mathrm{l}$ and $1 \mu \mathrm{l}$ of BDA, respectively. We have assumed that the pattern of connections revealed with BDA is that which is present at the time of perfusion (Simon and O'Leary, 1992).

Histochemical processing. After a postinjection survival period of 2 days, the animals were deeply anesthetized with pentobarbital sodium (100 mg/kg i.p.) and perfused through the heart with $0.9 \%$ saline followed by $2 \%$ paraformaldehyde in $0.1 \mathrm{M}$ phosphate buffer (PB, pH 7.4). The brains were removed, left overnight in $30 \%$ sucrose and $0.1 \mathrm{M} \mathrm{PB}$, and cut into $60 \mu \mathrm{m}$ thick coronal sections with a freezing microtome. BDA labeling was revealed using the standard Avidin-BiotinPeroxidase protocol (Vectastain Elite ABC kit, Vector Laboratories, Burlingame, CA) and $0.01 \% \mathrm{H}_{2} \mathrm{O} 2$ in $0.05 \% 3-3$, diaminobenzidine, with cobalt or nickel intensification; sections were then mounted, dehydrated, defatted, and coverslipped. Sections examined only for fluorescence were mounted without further processing and, after the data had been collected, they were Nissl-stained to reveal the location of area 17.

Data acquisition and analysis. In both MK-801 treated and control animals, the location of the injection sites with respect to the lateral border of striate cortex was determined according to architectonic criteria in sections stained for Nissl substance (Zilles et al., 1984), and by analyzing the distribution of labeled fields within the ipsilateral dorsal lateral geniculate nucleus (dLGN) of the thalamus (Montero et al., 1968). The distributions of cells labeled retrogradely and callosal axons labeled anterogradely were charted using a microscope equipped with a drawing tube and a motorized stage (LEPCO) controlled by a Dell XPS T500 computer running a graphics program (Neurolucida, MicroBrightField, Willistone, VT).

To analyze the morphology of callosal arbors, identified fibers extending through most of the cortical thickness were drawn using Neurolucida and a $40 \mathrm{X}$ objective. Branches measuring less than $15 \mu \mathrm{m}$ were 
A

CONTROL

SI 13
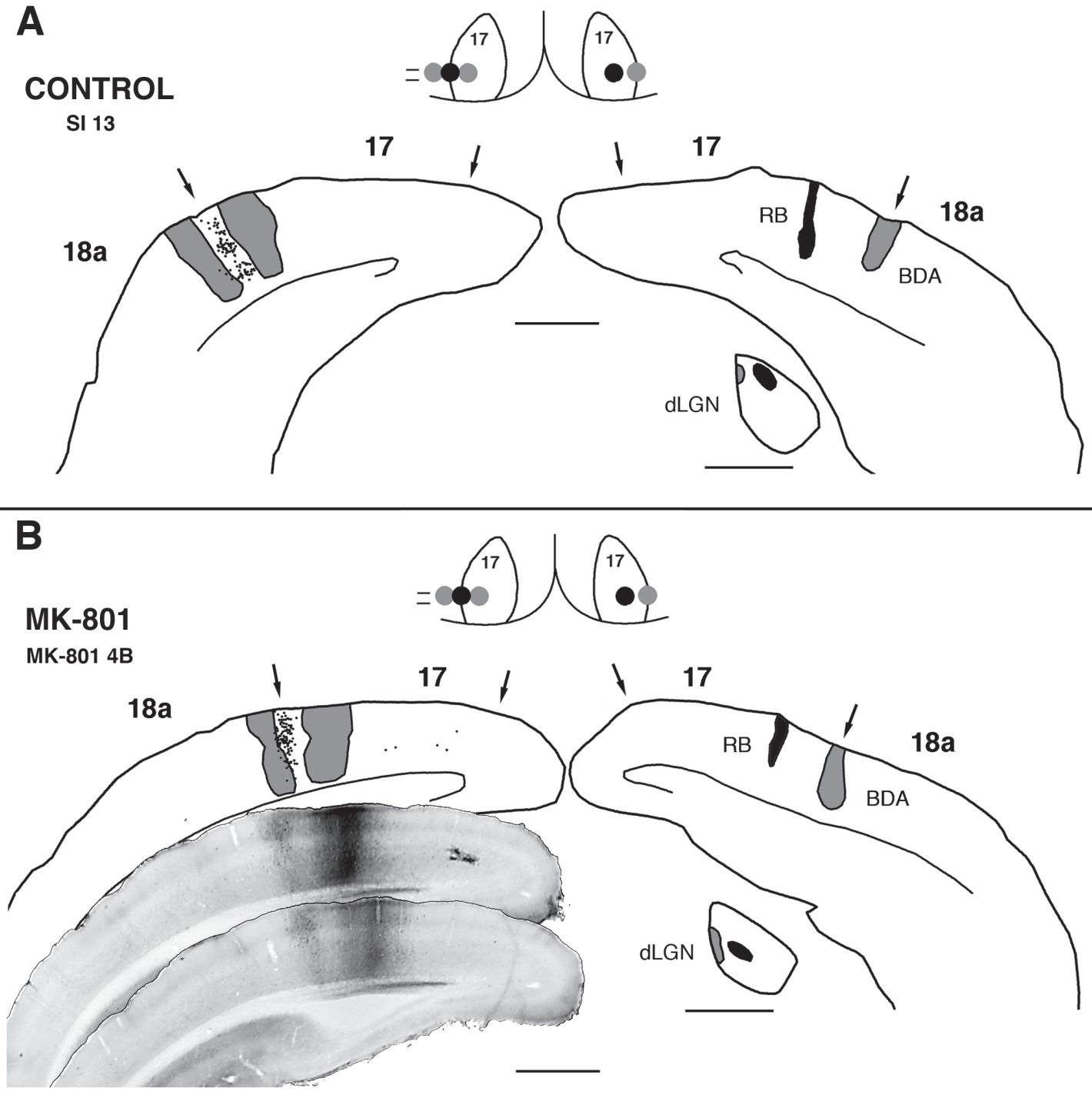

Fig. 2: Effect of MK-801treatment during the P4-P6 critical period on the topography of the callosal map. A: Organization of callosal linkages in the 17/18a callosal region of a control rat (case SI 13). This case received an injection of BDA at the 17/18a border (indicated in gray at right), and an injection of RB placed $1000 \mu \mathrm{m}$ more medially (indicated in black). Arrows indicate the medial and lateral borders of area 17. Drawing of left hemisphere shows the distribution of RB-labeled cells (black dots) and BDA-labeled fibers (gray areas) reconstructed from two sections taken from the region indicated by the parallel lines in the top inset. The results indicate that callosal projections in control rats were non-symmetric. B: Organization of callosal linkages in the $17 / 18$ a callosal region of adult rat injected with MK-801 from P4 to P8 (case MK-801 4B). This animal received an injection of BDA at the 17/18a border (indicated in gray at right), and an injection of RB placed $900 \mu \mathrm{m}$ more medially (indicated in black at right). Conventions are as in A. Drawing of left hemisphere shows the distribution of RB-labeled cells (black dots) and BDA-labeled fibers (gray) reconstructed from two sections taken from the region indicated by the parallel lines in the top inset. The BDA-labeled fields are shown in low power micrographs of two histological sections. Comparison of the results shown in A and B indicate that MK-801 treatment did not induce obvious changes in callosal topography. In both A and B, bottom right insets illustrate the location of the labeled fields in the dLGN (medial is to the left). Scale bars $=1.00 \mathrm{~mm}$. 
not included in the data. For each fiber, the following parameters were measured using the program ImageJ: 1) total arbor length, obtained by adding the length of all axonal branches; 2) number of terminal branches; and 3) average branch length, i.e., the ratio between the total arbor length and the number of branches. Statistical analysis was performed using the Student's t-test, with significance set at $p=0.05$. While some of the tips of the traced terminal branches had bouton-like endings, it is likely that some terminal branches were cut in the sectioning process. Although the absolute length of branches and number of terminal tips cannot therefore be determined, analysis of large numbers of axons makes it is possible to perform meaningful comparisons between arbors from experimental and control groups. A similar approach was used in previous studies of the development and plasticity of axonal arbors (e.g., Fish et al., 1991; Hedin-Pereira et al., 1999; Lee et al., 2005a). Figures were prepared using PhotoShop 9.02 (Adobe Systems, Mountain View, CA), and all image processing used, including contrast enhancement and intensity level adjustments, was applied to the entire image and never locally.

\section{RESULTS}

Effect of MK-801 on the topography of the callosal map

Our results from control rats, illustrated in Fig. 2A, confirm the organization of callosal linkages reported previously in normal rats (Lewis and Olavarría, 1995). The case shown in Fig. 2A received an injection of BDA at the 17/18a border, and an injection of $\mathrm{RB}$ placed $1000 \mu \mathrm{m}$ more medially. The injection of BDA (colored gray in right panel of Fig. 2A) produced densely labeled fields of anterogradely labeled callosal fibers located both immediately within and immediately outside the 17/18a border (labeled fields colored gray in left panel in Fig. 2A). In contrast, the injection of RB (colored black in Fig. 2A) produced a dense accumulation of retrogradely labeled callosal cells centered on the $17 / 18$ a border (black dots in left panel in Fig. 2A). When the locations of the fields located at the border or inside area 17 are considered, these results indicate that callosal linkages in area 17 of control rats are not symmetric with respect to the brain midline, as in normal rats (see top inset in Fig. 2A). Similar results were observed in two other control rats studied using the same approach (data not shown).

Figure 2B illustrates the results we obtained from 6 rats treated with MK-801 for 5 days starting at P3 or P4. Case MK$8014 \mathrm{~B}$ received an injection of BDA at the $17 / 18$ a border (indicated in gray on the right panel) and an injection of RB located $900 \mu \mathrm{m}$ more medially (indicated in black). The injection of BDA produced two dense fields of anterogradely labeled callosal fibers located on either side of the 17/18a border in the contralateral hemisphere (gray areas in left panel, Fig. 2B), while the injection of RB produced a dense accumulation of retrogradely labeled cells centered on the 17/18a border (black dots in left panel). These results, schematically represented in the top inset in Figure 2B, provide evidence that callosal linkages are not arranged as a mirror image of the injections sites, as it occurs in neonatally enucleated rats (Olavarría and Hiroi, 2003). Similar results were observed in 5 additional rats treated with MK-801. In particular, we found that interchanging the location of the injections of anterograde or retrograde tracers did not change the topography of the labeled connections (data not shown). From these data we conclude that in MK-801 treated rats, cells located on either side of the $17 / 18$ a border send a convergent projection to the contralateral $17 / 18$ a border, while cells located at the 17/ $18 \mathrm{a}$ border send projections to regions immediately lateral and medial to the 17/ 18 a border. This organization of callosal linkages closely resembles the topography we observed in control rats (Fig. 2A), as well as that described in lateral area 17 in normal adult rats (Lewis and Olavarría, 1995; Olavarría and Hiroi, 2003). These results indicate that blockade of NMDARs with MK-801 during the P4-P6 critical period does not change the topography of 
callosal connections in area 17 in an obvious way.

\section{Effect of MK-801 and neonatal enucleation on the architecture of callosal axon arbors}

To examine the effect of pharmacological blockade of NMDARs on the elaboration of callosal arbors, MK-801 was administered for 5 days starting at P6, when invasion of fibers into upper layers of gray matter and elaboration of arbors are just beginning (Olavarría and Safaeian, 2006). Callosal arbors were revealed with BDA injections in adult rats. Figure $3 \mathrm{~A}$ illustrates drawings from a control rat (left panel) and a rat treated with MK-801 (right panel). Comparison of these drawings indicates that the arbor in the MK-801 treated rat is larger than that in the control animal. To quantify these observations, we measured the branch length, total arbor length and number of branches in 95 arbors drawn from 3 control rats and 82 arbors from 3 MK-801 treated rats. We found that branch length (Fig. 3B, Control $=89.5 \pm 12.9 \mu \mathrm{m}$; MK-801 = $120 \pm 11.7 \mu \mathrm{m})$ and total arbor length (Fig. 3C, Control $=271.8 \pm 27.8 \mu \mathrm{m}$; MK-801 = 427.6 $\pm 43.5 \mu \mathrm{m})$ were significantly larger $(\mathrm{p}<0.05)$ in MK-801 treated than in control rats, but we observed no significant difference in the number of branches between these two groups of animals (average of $3.1 \pm 0.23$ branches in control rats, and $3.75 \pm 0.75$ branches in MK-801 treated rats).

We also studied arbor architecture in both normal and neonatally enucleated rats at early stages of development. The left panel in Figure 4A shows the architecture of a callosal axon at P6 in a normal pup, confirming previous reports that at this age many callosal axons reach upper layers of gray matter and usually display few, short branches (Fish et al., 1991, Norris and Kalil, 1992; Hedin-Pereira et al., 1999;
A

CONTROL

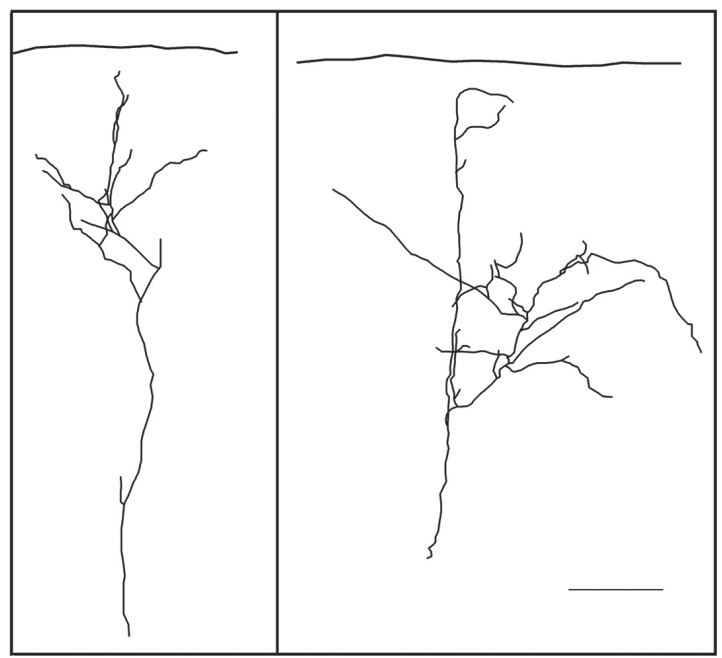

B

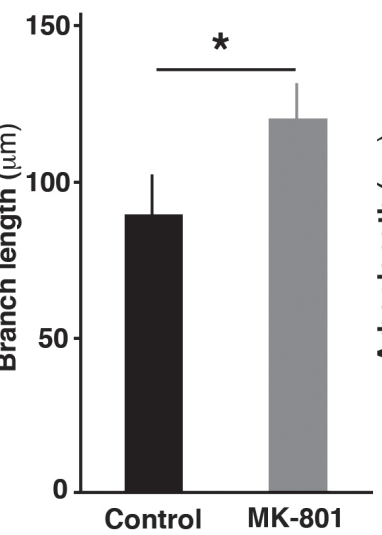

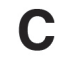

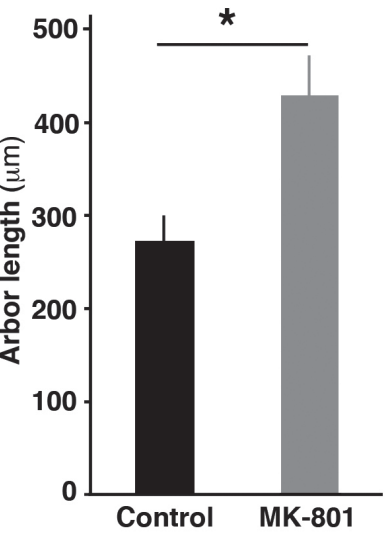

Fig. 3: Effects of MK-801treatment started at P6 on the architecture of callosal arbors. A: Representative drawings of arbors taken from control (left) and MK-801 (right) treated rats. Line at top indicates the pial surface. Scale bar $=1.0 \mathrm{~mm}$. B: Histogram compares the mean values for branch length in 94 arbors from 3 control rats (black bar) and in 82 arbors from 3 MK-801 treated (gray bar) rats. The asterisk indicates that branch length in MK-801 treated rats $(120 \pm 11.7 \mu \mathrm{m})$ was significantly larger $(\mathrm{p}<0.05)$ than in control rats $(89.5 \pm 12.9 \mu \mathrm{m})$. C: Comparison of mean values for total arbor length measured in the same arbors analyzed in B. The asterisk indicates that total arbor length in MK-801 treated rats $(427.6 \pm 43.5 \mu \mathrm{m})$ was significantly larger $(\mathrm{p}<0.05)$ than in control rats $(271.8 \pm 27.8 \mu \mathrm{m})$. Means and STDV are graphed in B and C. 
Ding and Elberger, 2001; Olavarría and Safaeian, 2006). The right panel in Figure 4A illustrates our finding that neonatal enucleation increases the size of callosal arbors, and that this effect is present at early stages of development. We measured the branch length, total arbor length and number of branches in 132 arbors drawn from 4 normal pups studied at P6-P8 and 127 arbors from 4 enucleated pups studied at the same ages. We found that branch length (Fig. 4B, N P6-P8 $=100.8 \pm 18.6$ $\mu \mathrm{m}$; BE0 P6-P8 $=134.0 \pm 14.6 \mu \mathrm{m}$ ) and total arbor length (Fig. 4C, N P6-P8 = 294 $\pm 78.8 \mu \mathrm{m}$; BE0 P6-P8 = $487.0 \pm 113.0 \mu \mathrm{m})$ were significantly larger $(\mathrm{p}<0.05)$ in enucleated pups than in control pups, but there was no significant difference in the number of branches between these two groups of animals (average of $2.5 \pm 0.29$ branches in control rats, and $3.35 \pm 0.69$ branches in MK-801 treated rats).

\section{DISCUSSION}

To investigate whether retinal influences on callosal topography are mediated by NMDARs, we studied the topography of callosal linkages in adult rats that had been injected with the NMDAR blocker MK-801 during the P4-P6 critical period. We expected that blockade of NMDARs during this critical period would lead to the development of mirror-symmetric callosal linkages, thus replicating the effect of removing retinal input at $\mathrm{P} 4$ (Olavarría and Hiroi, 2003). Instead, we found that
A

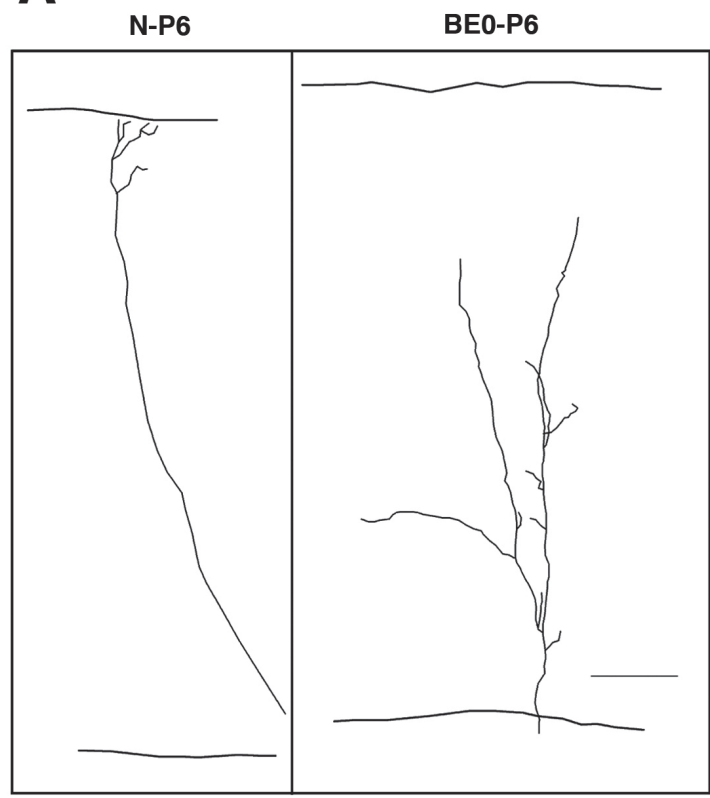

\section{B}

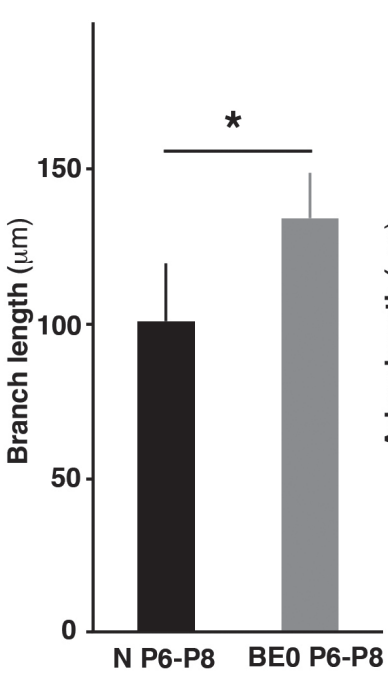

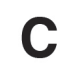

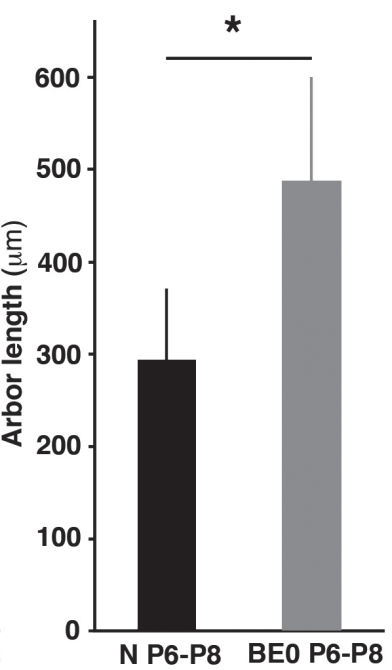

Fig. 4: Effect of neonatal bilateral enucleation on the development or callosal arbors. A: Representative drawings of arbors taken from a P6 control rat (N-P6, left) and from a P6 rat enucleated at birth (BE0-P6, right). Lines at top and bottom indicate pial surface and white matter, respectively. Scale bar $=1.0 \mathrm{~mm}$. B: Histogram compares the mean values for branch length in 132 arbors from 4 control pups studied at ages P6-P8 (black bar) and in 127 arbors from 4 enucleated pups studied at the same ages (P6-P8, gray bar). The asterisk indicates that branch length in enucleated rats $(134 \pm 14.6 \mu \mathrm{m})$ was significantly larger $(\mathrm{p}<0.05)$ than in control rats $(100.8 \pm 18.6$ $\mu \mathrm{m})$. C: Comparison of mean values for total arbor length measured in the same arbors analyzed in B. The asterisk indicates that total arbor length in enucleated rats $(487.0 \pm 113.0 \mu \mathrm{m})$ was significantly larger $(\mathrm{p}<0.05)$ than in control rats $(294 \pm 78.8 \mu \mathrm{m})$. Means and STDV are graphed in $\mathrm{B}$ and $\mathrm{C}$. 
pharmacological blockade of NMDARs from P4-P6 did not induce obvious abnormalities in the topography of callosal linkages: callosal linkages were non-mirror symmetric, as in control rats. These results provide evidence that the influences that the eyes exert on callosal topography during the P4-P6 critical period do not operate through NMDAR-mediated processes. In contrast, we found that interfering with NMDAR function either through MK801induced blockade of NMDARs starting at P6 or neonatal enucleation significantly increases the length of axon branches and total length of arbors, without major effects on the number of branch tips.

It is unlikely that our injections of MK801 did not adequately block NMDARs because we used dosages that were similar or larger than those used in some previous studies (e.g., Wilson et al., 1998; Daw et al., 1999). The effects of MK-801 injections on the motility and responsiveness of our pups were similar to those described in studies in which the effects of MK-801 on neuronal activity were evaluated electrophysiologically (Daw et al., 1999). In addition, we found that equivalent dosages administered to animals P6 or older did have an effect on the development of callosal arbor architecture.

It is possible that the administration regime we used to block NMDARs with MK-801 did not significantly reduce spontaneous activity along the visual pathway (Daw et al., 1999). This scenario leaves open the possibility that callosal topography may depend on activity mediated by receptors other than NMDARs. Alternatively, it is possible that spontaneous activity was significantly depressed in animals treated with MK-801, implying that spontaneous activity does not play an important role in the specification of the callosal map (see Chang et al., 1995). Indeed, bilateral projections from temporal retina may guide callosal development, not by means of activity-dependent cues, but by relaying chemical labels that lead to the establishment of retinotopically matched callosal linkages (Chang et al., 1995). As discussed in Olavarría and Hiroi (2003), retinal input during the P4-P6 critical period may set in motion a master mechanism that triggers multiple effects along the visual pathway, including changes in the kinetics of NMDAR-mediated currents (Olavarría et al., 2007), as well as in the expression or activation of various signaling molecules (see Olavarría and Hiroi, 2003, for Refs.). While the expression/activation of signaling pathways may be responsible for the specification of the callosal blueprint by P6, other effects, such as the increase in the duration of NMDAR-mediated currents that occurs during P6-P13, may influence the subsequent elaboration of axonal arbors and dendrites.

The idea that NMDARs influence the development of axonal arbors and dendrites is supported by studies showing that NMDARs regulate the growth of presynaptic terminal arbors and postsynaptic dendritic branching in several systems (Brewer and Cotman, 1989; Schmidt et al., 2000; Lee et al., 2005a,b). Lee et al., (2005a) studied cortex-specific NR1 knock-out mice and found that thalamocortical afferents develop far more extensive arbors than the arbors in control animals. In another study, Lee et al. (2005b) found that in NR1 gene knockdown and knock-out mice, whisker afferents in the trigeminal principal nucleus begin their development normally but subsequently develop exuberant terminal arbors. They also reported that barrelette cells in the trigeminal principal nucleus develop longer dendrites with no orientation preference in NR1 gene knockdown mice. Schmidt et al. (2000) found that MK-801 significantly enlarged the size of retinotectal arbors in zebrafish. In frogs, Cline and Constantine-Paton (1989) found that retinotectal arbors were significantly elongated after application of the NMDAR blocker AP5. Similarly, in ferrets, Hahm et al. (1991) reported that retinal arbors in the dLGN were enlarged after MK-801 treatment. In agreement with these studies, we found that in adult rats injected with MK-801 from P6 to P10, the average length of branches and the total length of arbors were significantly greater than in control adult animals. Also in agreement with 
Schmidt et al. (2000) and Lee et al. (2005a,b), we found no significant difference in the number of branch tips. Given that NMDARs are spared in cortically projecting thalamic cells of cortex-restricted NR1 knock-out mice, Lee et al (2005a) concluded that postsynaptic NMDARs play an important role in the refinement of presynaptic thalamocortical afferent arbors in the barrel cortex.

In neonatally enucleated rats studied at P6-P8 we found that the average length of branches and the total length of arbors were significantly greater than in normal animals of the same age. These observations are in agreement with studies showing that neonatal infraorbital nerve cut results in larger thalamocortical arbors in somatosensory cortex (Jensen and Killackey, 1987). Also as in the somatosensory system (Catalano et al., 1995), the effects of neonatal enucleation on callosal axons were rapid, indicating that deafferentation in both the visual and somatosensory systems acts by altering the initial elaboration of arbors in the neocortex rather than by inhibiting a later-occurring remodeling process. The report that adult hamsters enucleated at birth have callosal arbors that are more widespread than normal (Fish et al., 1991) indicates that at least some of the early effects of enucleation on arbor development persist into adulthood. In agreement with Fish et al. (1991), we found that neonatal enucleation increases the length of branches without significantly increasing the number of branches.

The observation that the effects of enucleation resemble those observed in MK-801 treated rats (present study) and NR1 deficient mice (Schmidt et al., 2000; Lee et al., 2005a,b) supports the idea that enucleation affects arbor elaboration by interfering with the function of NMDARs. It is possible that enucleation leads to a reduction in NMDAR activation due to either reduced cortical spontaneous activity or asynchrony between callosal afferents and cortical neurons, and that this reduction in NMDAR activation is the primary reason for longer branches in enucleated animals. Alternatively, enucleation at birth may interfere with arbor development because of its effects on the duration of NMDARmediated responses. By $\mathrm{P} 6$, when invasion of supragranular layers and elaboration of arbors begin (Fish et al., 1991, Norris and Kalil, 1992; Hedin-Pereira et al., 1999; Ding and Elberger, 2001; Olavarría and Safaeian, 2006), the eyes induce a lengthening of the synaptic response mediated by NMDARs in callosal cells (Olavarría et al., 2007). However, bilateral enucleation at or before $\mathrm{P} 4$, but not at or after P6, abolishes this transient increase in the duration of NMDAR-mediated responses (Olavarría et al., 2007). The idea that reductions in NMDAR-mediated synaptic currents may affect arbor development is consistent with the report that barrelette cells in the trigeminal principal nucleus of NR1 gene knock-down mice show significantly reduced NMDAR currents and develop longer dendrites with no orientation preference (Lee et al., $2005 b)$. It is therefore possible that the role of NMDAR in arbor architecture depends at least in part on the duration of NMDAR currents. Slow NMDAR synaptic responses increase inflow of $\mathrm{Ca}^{2+}$, which may trigger or enhance metabolic pathways involved in the regulation of arbor elaboration (reviewed in Lee et al., 2005b). In contrast, shortening of NMDAR-mediated responses during the period of arbor elaboration may interfere with processes regulating arbor growth, leading to larger arbors. By regulating internal $\mathrm{Ca}^{2+}$ levels (Scatton, 1993), NMDARs could affect a number of downstream signal transduction pathways involved in neuron architecture. For example, the activity of $\mathrm{Ca}^{2+} /$ calmodulindependent protein kinase II (CaMKII) is regulated by $\mathrm{Ca}^{2+}$ influx through NMDARs (Colbran, 1992), and CaMKII has been implicated in refinement of retinal connections (Zou and Cline, 1999). It has also been suggested that deficiencies in NMDAR function may cause growing axons to ignore stop signals resulting in abnormally large arbors (reviewed in Lee et al., 2005a).

In conclusion, results from all experiments in this study indicate that while activity mediated by NMDARs is not 
necessary during P4-P6 for the specification of the callosal map, NMDARs appear to play a role in the subsequent development of callosal arbors. These observations suggest that development of callosal projections is influenced by different mechanisms acting at specific postnatal ages. Our findings further suggest that NMDAR-mediated processes shape arbor architecture primarily by regulating the initial elaboration of arbors in the neocortex rather than by promoting a later-occurring pruning process. A previous study showed that callosal topography is present by P6, just as axonal branches of simple architecture grow into superficial cortical layers (Olavarría and Safaeian, 2006). If arbors develop in an exuberant fashion, this initial topography could be blurred or abolished altogether at later stages of development. Our present results therefore raise the possibility that regulation of arbor development by NMDAR-mediated processes may be important for maintaining the precision of cortical maps during the period of arbor elaboration and later in life.

\section{ACKNOWLEDGMENTS}

This work was supported in part by a RRF award, and National Institutes of Health grant number: EY016045.

\section{REFERENCES}

BREWER GJ, COTMAN CW (1989) NMDA receptor regulation of neuronal morphology in cultured hippocampal neurons. Neurosci Lett 99: 268-273.

CATALANO SM, ROBERTSON RT, KILLACKEY HP (1995) Rapid alteration of thalamocortical axon morphology follows peripheral damage in the neonatal rat. Proc Natl Acad Sci 92: 2549-52.

CHANG K, VAN SLUYTERS RC, OLAVARRÍA J (1995) Effect of monocular blockade of retinal activity on the development of visual callosal connections in the rat. Biol Res 28: 219-226.

CLINE HT, CONSTANTINE-PATON M (1989) NMDA receptor antagonists disrupt the retinotectal topographic map. Neuron 3: 413- 426.

COLBRAN RJ (1992) Regulation and role of brain calcium/calmodulin-dependent protein kinase II. Neurochem Int 21: 469-497

CONSTANTINE-PATON M, CLINE HT, DEBSKI E (1990) Patterned activity, synaptic convergence, and the NMDA receptor in developing visual pathways. Annu Rev Neurosci 13: 129-154
DAW NW, GORDON B, FOX KD, FLAVIN HJ, KIRSCH JD, BEAVER CJ, JI Q -H, REID SNM, CZEPITA D (1999) Injection of MK-801 affects ocular dominance shifts more than visual activity. J Neurophysiol 81: 204-215.

DING S-L, ELBERGER AJ (2001) Postnatal development of biotinylated dextran amine-labeled corpus callosum axons projecting from the visual and auditory cortices to the visual cortex of the rat. Exp Brain Res 136: 179193.

FISH SE, RHOADES RW, BENNETT-CLARKE CA, FIGLEY B, MOONEY RD (1991) Organization, development and enucleation-induced alterations in the visual callosal projection of the hamster: single axon tracing with Phaseolus vulgaris leucoagglutinin and Di-I. Eur J Neurosci 3: 1255-1270.

HAHM JO, LANGDON RB, SUR M (1991) Disruption of retinogeniculate afferent segregation by antagonists to NMDA receptors. Nature 351: $568-570$.

HEDIN-PEREIRA C, LENT R, JHAVERI S (1999) Morphogenesis of callosal arbors in the parietal cortex of hamsters. Cereb Cortex 9: 50-64.

HEBB DO (1949) The Organization of Behavior. New York: Wiley.

JENSEN K, KILLACKEY H P (1987) Terminal arbors of axons projecting to the somatosensory cortex of the adult rat. II. The altered morphology of thalamocortical afferents following neonatal infraorbital nerve cut. $\mathbf{J}$ Neurosci 7: 3544-3553

KATZ LC, SHATZ CJ (1996) Synaptic activity and the construction of cortical circuits. Science 274: 11331138.

LEE L-J, IWASATO T, ITOHARA S, ERZURUMLU RS (2005a) Exuberant thalamocortical axon arborization in cortex-specific nmdar1 knockout mice. J Comp Neurol 485: 280-292.

LEE L-J, LO F-S, ERZURUMLU RS (2005b) NMDA Receptor-Dependent Regulation of Axonal and Dendritic Branching. J Neurosci 25: 2304 -2311.

LEWIS JW, OLAVARRÍA JF (1995) Two rules for callosal connectivity in striate cortex of the rat. J Comp Neurol 361: 119-137.

MACDONALD JF, BARTLETT MC, MODY I, PAHAPILL P, REYNOLDS N, SALTER MW, SCHNEIDERMAN JH, PENNEFATHER PS (1991) Actions of ketamine, phencyclidine and MK-801 on NMDA receptor blockade currents in cultured mouse hippocampal neurons. J Physiol, London 432: 483-508.

MAFFEI L, GALLI-RESTA L (1990) Correlation in the discharges of neighboring rat retinal ganglion cells during prenatal life. Proc Natl Acad Sci USA 87: 28612864.

MONTERO VM, BRUGGE JF, BEITEL RE (1968) Relation of the visual field to the lateral geniculate body of the albino rat. J Neurophysiol 31: 221-136.

NORRIS CR, KALIL K (1992) Development of callosal connections in the sensorimotor cortex of the hamster. J Comp Neurol 326: 121-132.

OLAVARRÍA JF (2002) Influence of topography and ocular dominance on the functional organization of callosal connections in cat striate cortex. In: PAYNE B, PETERS A (eds) The cat primary visual cortex. New York: Academic Press. pp: 259-294.

OLAVARRÍA JF, HIROI R (2003) Retinal influences specify cortico-cortical maps by postnatal day six in rats and mice. J Comp Neurol 459: 156-172.

OLAVARRÍA JF, LI CH-P (1995) Effects of neonatal enucleation on the organization of callosal linkages in striate cortex of the rat. J Comp Neurol 361: 138-151.

OLAVARRÍA JF, SAFAEIAN P (2006) Development of 
callosal topography in visual cortex of normal and enucleated rats. J Comp Neurol 496: 495-512.

OLAVARRÍA JF, VAN BREDERODE JFM, SPAIN WJ (2007) Retinal influences induce bidirectional changes in the kinetics of $n$-methyl-d-aspartate receptormediated responses in striate cortex cells during postnatal development. Neurosci 148: 683-699.

SCHMIDT JT, BUZZARD M, BORRESS R, DHILLON S (2000) MK801 increases retinotectal arbor size in developing zebrafish without affecting kinetics of branch elimination and addition. J Neurobiol 42: 303314.

SCATTON B (1993) The NMDA receptor complex. Fundam Clin Pharmacol 7: 389-400.

SIMON DK, O'LEARY DDM (1992) Development of topographic order in the mammalian retinocollicular projection. J Neurosci 12: 1212-1232.

WILSON MA, KINSMAN SL, JOHNSTON MV (1998)

Expression of NMDA receptor subunit mRNA after MK-801 treatment in neonatal rats. Dev Brain Res 109: 211-220.

ZILLES K, WREE A, SCHLEICHER A, DIVAC I (1984) The monocular and binocular subfields of the rat's primary visual cortex: a quantitative morphological approach. J Comp Neurol 226: 391-402.

ZOU DJ, CLINE HT (1999) Postsynaptic calcium/ calmodulin-dependent protein kinase II is required to limit elaboration of presynaptic and postsynaptic neuronal arbors. J Neurosci 19: 8909-8918. 